\title{
Análise Comparativa Entre Modelos Dinâmicos Aplicados ao Rastreamento de Objetos Manobrantes *
}

\author{
Sarah Beatriz Gruetzmacher* Pedro Humberto Nunes* \\ Victor Baptista Frencl* \\ * Departamento Acadêmico de Eletrotécnica, Universidade Tecnológica \\ Federal do Paraná, PR, (e-mails: $\{$ sarah, \\ pedronunes\}@alunos.utfpr.edu.br,vbfrencl@utfpr.edu.br).
}

\begin{abstract}
In object tracking problems, one of the concerns is the choice of the mathematical model that represents the tracking system, especially the object's dynamics. In the literature, there are several dynamic models (cartesian and polar) that can be used to represent the object maneuvers. However, some of these dynamic models represent only two-dimensional movements. This paper has two main objectives: restructure some of the two-dimensional polar dynamic models found in the literature, in such a way to represent maneuvering objects trajectories in a three-dimensional space, and compare these restructured dynamic models with a threedimensional cartesian dynamic model from the literature. In order to verify if the restructured models are suitable, they will be implemented computationally in an extended Kalman filter. Mean squared errors based on estimations and code runtime will be used as performance criteria. For the trajectories measurements, it will be used simulated data from a trajectory generator software.

Resumo: Em problemas de rastreamento de objetos, uma das preocupações é a escolha do modelo matemático que represente o sistema de rastreamento, especialmente a dinâmica do objeto. Na literatura, existem diversos modelos dinâmicos (cartesianos e polares) que podem ser utilizados para representar as manobras do objeto. Entretanto, uma parte destes modelos dinâmicos representa apenas movimentações bidimensionais. Este artigo tem dois objetivos principais: reestruturar alguns modelos dinâmicos polares bidimensionais encontrados na literatura, de maneira a fazer com que representem trajetórias de objetos manobrantes no espaço tridimensional, e comparar tais modelos dinâmicos reestruturados com um modelo dinâmico cartesiano tridimensional da literatura. Para verificar se tais modelos são adequados, estes serão implementados computacionalmente em um filtro de Kalman estendido. Erros quadráticos médios de estimativas e o tempo de execução do código serão utilizados como critérios de desempenho. Para as medidas das trajetórias serão utilizados dados simulados a partir de um software gerador de trajetórias.
\end{abstract}

Keywords: Object tracking; dynamic models; maneuvering objects; stochastic filtering; extended Kalman filter.

Palavras-chaves: Rastreamento de objetos; modelos dinâmicos; objetos manobrantes; filtragem estocástica; filtro de Kalman estendido.

\section{INTRODUÇÃO}

Em problemas de rastreamento de objetos, uma das etapas fundamentais é a obtenção de estimativas de estado, via filtragem estocástica, a partir de medições ruidosas capturadas por sensores como radares, sonares, entre outros. Para que a qualidade destas estimativas seja considerada boa, é necessário que o modelo matemático da dinâmica do objeto represente seus movimentos da melhor maneira possível.

Em (Rong Li and Jilkov, 2003), apresenta-se um estudo feito em relação a alguns modelos dinâmicos cartesia-

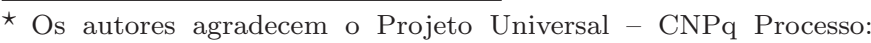
421486/2016-3 pelo suporte financeiro.
}

nos que representam um objeto em movimento, tanto no plano - modelos bidimensionais - quanto no espaço - modelos tridimensionais. Dentre os modelos dinâmicos apresentados, os modelos mais frequentemente utilizados no rastreamento de objetos são os modelos de velocidade constante (VC), os modelos de aceleração constante (AC) e os modelos de giro constante (GC). Alguns desses modelos podem ser encontrados na literatura com a sua estrutura modificada, dependendo da aplicação desejada ou do problema a ser tratado.

Em (Frencl et al., 2008), é proposto um modelo GC cartesiano tridimensional, remodelado de maneira a incluir a velocidade angular como um dos elementos do vetor de estados. Em (Gustafsson and Isaksson, 1996), propõem- 
se um modelo GC bidimensional que lida com parâmetros no formato polar para definição da velocidade do objeto. Em (Kang et al., 2017), é feita uma modificação em um modelo GC em coordenadas polares, em que se considera a velocidade angular não como um estado, mas como um dos parâmetros externos de controle, o que também acontece com a aceleração.

É importante notar que boa parte dos modelos dinâmicos encontrados na literatura baseiam-se em objetos que se movem bidimensionalmente. Porém, é bastante comum encontrar objetos que se movem no espaço tridimensional, como aeronaves e drones. Desta forma, este trabalho se propõe, primeiramente, a realizar alterações em alguns modelos GC bidimensionais polares da literatura, de tal maneira a redefini-los para modelos GC tridimensionais polares. Outra proposta deste trabalho é comparar a qualidade das estimativas resultantes de um filtro estocástico, quando se utilizam os modelos dinâmicos tridimensionais polares obtidos neste trabalho e quando se utiliza o modelo cartesiano tridimensional proposto em (Frencl et al., 2008).

Para realizar as comparações entre os modelos dinâmicos, estes serão implementados computacionalmente em um Filtro de Kalman Estendido (FKE) (Bar-Shalom et al., 2001), que lida com modelos matemáticos não lineares. Este comparativo será baseado em dois critérios de desempenho comumente utilizados: Erros Quadráticos Médios (EQM) das estimativas e o tempo de execução do código. As medidas a serem utilizadas no FKE são obtidas a partir de simulações do gerador de trajetórias, proposto em (Martins et al., 2018). Algumas modificações neste gerador foram feitas para que parâmetros de aceleração normal e tangencial pudessem ser incluídos como novos parâmetros.

\section{MODELOS MATEMÁtiCOS}

Modelos matemáticos de sistemas podem ser representados de diversas formas (Ogata, 2015). Em problemas que envolvem a utilização de filtros estocásticos, estes modelos devem ser representados em espaço de estados. Além disso, estes modelos devem levar em conta eventuais imprecisões na dinâmica e nas medidas, devido a perturbações e distúrbios, internos e externos ao sistema (Jazwinski, 1970).

Tal modelo matemático é descrito pelas seguintes equações a tempo discreto, com $k \in \mathbb{N}$ :

$$
\begin{aligned}
& \mathbf{x}_{k}=\mathbf{f}\left(\mathbf{x}_{k-1}, \mathbf{u}_{k-1}\right)+G_{k-1} \mathbf{w}_{k-1}, \\
& \mathbf{y}_{k}=\mathbf{g}\left(\mathbf{x}_{k}, \mathbf{u}_{k}\right)+\mathbf{v}_{k},
\end{aligned}
$$

em que $\mathbf{x}_{k} \in \mathbb{R}^{n}$ é o vetor de estados, $\mathbf{u}_{k} \in \mathbb{R}^{r}$ é o vetor de controle (termo determinístico), $\mathbf{y}_{k} \in \mathbb{R}^{m}$ é o vetor de medidas, $\mathbf{w}_{k} \in \mathbb{R}^{n_{w}}$ é o ruído do processo (termo estocástico), cuja distribuição é gaussiana, com média nula e matriz de covariância $Q_{k} \in \mathbb{R}^{n \times n}, \mathbf{v}_{k} \in \mathbb{R}^{m_{v}}$ é o ruído de medida (termo estocástico), cuja distribuição também é gaussiana, com média nula e matriz de covariância $R_{k} \in$ $\mathbb{R}^{m \times m}, G_{k} \in \mathbb{R}^{n \times n_{w}}$ e $\mathbf{f}(\cdot)$ e $\mathbf{g}(\cdot)$ são funções vetoriais, lineares ou não.

Para todos os casos tratados aqui neste artigo, o vetor de medidas $\mathbf{y}_{k}$ será descrito por:

$$
\mathbf{y}_{k}=\left[\begin{array}{lll}
p_{x} & p_{y} & p_{z}
\end{array}\right]^{\top},
$$

em que $p_{x}$ é a posição do objeto na coordenada $x, p_{y}$ é a posição do objeto na coordenada $y$ e $p_{z}$ é a posição do objeto na coordenada $z$. Como os vetores de estado tratados neste trabalho terão, pelo menos, as posições $p_{x}$, $p_{y}$ e $p_{z}$ como seus elementos, a função $\mathbf{g}(\cdot)$ sempre será considerada linear. Por exemplo, se $\mathbf{x}_{k}=\left[\begin{array}{llll}p_{x} & p_{y} & p_{z} & \omega\end{array}\right]^{\top}$, a função $\mathbf{g}(\cdot)$ será dada por:

$$
\mathbf{g}\left(\mathbf{x}_{k}\right)=\left[\begin{array}{llll}
1 & 0 & 0 & 0 \\
0 & 1 & 0 & 0 \\
0 & 0 & 1 & 0
\end{array}\right] \mathbf{x}_{k} .
$$

O interesse principal deste trabalho está em tratar a função $\mathbf{f}(\cdot)$, que define a dinâmica do sistema, já que um dos objetivos é adaptar modelos dinâmicos bidimensionais encontrados na literatura, de tal maneira que esses modelos representem manobras tridimensionais no espaço.

\section{MODELOS DINÂMICOS}

Nas seções a seguir, serão apresentadas as formulações matemáticas de três modelos dinâmicos de interesse deste trabalho, sendo um deles um modelo cartesiano tridimensional e outros dois modelos polares bidimensionais, que serão reestruturados para se tornarem modelos polares tridimensionais. Esta reestruturação baseia-se no aumento dos vetores de estado com novos elementos e, consequentemente, no aumento das matrizes que definem a dinâmica do objeto.

\subsection{Modelo GC com Velocidade Cartesiana}

Em (Frencl et al., 2008), é proposto um modelo GC tridimensional com velocidade cartesiana (GCVC), em que o objeto está realizando uma trajetória curva no espaço. Como o valor da velocidade angular $\omega$ raramente é conhecido em rastreamento de objetos, tal variável é adicionada ao vetor de estados original, gerando um vetor de estados aumentado, dado por:

$$
\mathbf{x}_{k}=\left[\begin{array}{lllllll}
p_{x} & v_{x} & p_{y} & v_{y} & p_{z} & v_{z} & \omega
\end{array}\right]_{k}^{\top}
$$

em que $v_{x}$ é a velocidade do objeto na direção da coordenada $x, v_{y}$ é a velocidade do objeto na direção da coordenada $y, v_{z}$ é a velocidade do objeto na direção da coordenada $z$ e $\omega$ é a velocidade angular do objeto.

Com base em (1), a equação do espaço de estados para o modelo dinâmico em questão pode ser reescrita da seguinte maneira:

$$
\mathbf{x}_{k}=F_{k-1} \mathbf{x}_{k-1}+G_{k-1} \mathbf{w}_{k-1}
$$

em que:

$$
\begin{aligned}
& F_{k}=\left[\begin{array}{cc}
F_{x y} & \mathbf{0}_{4 \times 3} \\
\mathbf{0}_{3 \times 4} & F_{0}
\end{array}\right], \\
& F_{x y}=\left[\begin{array}{cccc}
1 & \frac{\operatorname{sen}(\omega T)}{\omega} & 0 & -\frac{1-\cos (\omega T)}{\omega} \\
0 & \cos (\omega T) & 0 & -\operatorname{sen}(\omega T) \\
0 & \frac{1-\cos (\omega T)}{\omega} & 1 & \frac{\operatorname{sen}(\omega T)}{\omega} \\
0 & \operatorname{sen}(\omega T) & 0 & \cos (\omega T)
\end{array}\right]_{k} \\
& F_{0}=\left[\begin{array}{lll}
1 & T & 0 \\
0 & 1 & 0 \\
0 & 0 & 1
\end{array}\right] \text {, } \\
& G_{k}=\left[\begin{array}{lllllll}
0 & 1 & 0 & 0 & 0 & 0 & 0 \\
0 & 0 & 0 & 1 & 0 & 0 & 0 \\
0 & 0 & 0 & 0 & 0 & 1 & 0 \\
0 & 0 & 0 & 0 & 0 & 0 & 1
\end{array}\right]^{\top}
\end{aligned}
$$


$T$ é o período de amostragem e $\mathbf{0}_{i \times j} \in \mathbb{R}^{i \times j}$ é uma matriz preenchida com zeros. Note que, dada a estrutura da matriz $G_{k}$, os ruídos de processo afetam apenas os elementos de velocidade cartesiana e angular.

\subsection{Modelo GC com Velocidade Polar}

O modelo GC com velocidade polar (GCVP), proposto em (Gustafsson and Isaksson, 1996), utiliza o módulo da velocidade $v$ e seu ângulo de rumo $\theta$ para definir a velocidade do alvo, ao invés de utilizar as componentes de velocidade $v_{x}$ e $v_{y}$ como estados de $\mathbf{x}_{k}$. A velocidade angular $\omega$ também está presente em $\mathbf{x}_{k}$ como um dos estados. O vetor de estados para este modelo é:

$$
\mathbf{x}_{k}=\left[\begin{array}{lllll}
p_{x} & p_{y} & v & \theta & \omega
\end{array}\right]_{k}^{\top} .
$$

Para este modelo, a equação da dinâmica em (1) pode ser reescrita como:

$$
\mathbf{x}_{k}=\mathbf{f}\left(\mathbf{x}_{k-1}\right)+G_{k-1} \mathbf{w}_{k-1}
$$

em que

$$
\begin{aligned}
& \mathbf{f}\left(\mathbf{x}_{k}\right)=\left[\begin{array}{c}
p_{x}+\frac{2 v}{\omega} \operatorname{sen}\left(\frac{\omega T}{2}\right) \cos \left(\theta+\frac{\omega T}{2}\right) \\
p_{y}+\frac{2 v}{\omega} \operatorname{sen}\left(\frac{\omega T}{2}\right) \operatorname{sen}\left(\theta+\frac{\omega T}{2}\right) \\
v \\
\theta+\omega T \\
\omega
\end{array}\right]_{k}, \\
& G_{k}=\left[\begin{array}{ccccc}
0 & 0 & T^{2} & 0 & 0 \\
0 & 0 & 0 & \frac{T^{2}}{2} & T^{2}
\end{array}\right]^{\top} \text {. }
\end{aligned}
$$

Note que, para este modelo, não se consideram entradas externas $\left(\mathbf{u}_{k}=\mathbf{0}\right), \mathbf{f}(\cdot)$ é uma função não linear em relação às componentes do vetor de estados $\mathbf{x}_{k}$ e o vetor $\mathbf{w}_{k}$ é dado por $\mathbf{w}_{k}=\left[\begin{array}{ll}w_{v} & w_{\omega}\end{array}\right]_{k}^{\top}$.

Para reestruturar tal modelo para um formato tridimensional, supõe-se que a dinâmica na direção do eixo z é considerada de velocidade constante. Dessa forma, o vetor de estados aumentado fica da seguinte maneira:

$$
\mathbf{x}_{k}=\left[\begin{array}{lllllll}
p_{x} & p_{y} & p_{z} & v & \theta & \phi & \omega
\end{array}\right]^{\top},
$$

em que $\phi$ é o angulo de elevação do vetor velocidade em relação ao plano $x y$.

A partir das relações trigonométricas ligadas a coordenadas esféricas, é possível obter a função f(·) em (16).

$$
\mathbf{f}\left(\mathbf{x}_{k}\right)=\left[\begin{array}{c}
p_{x}+\frac{2 v}{\omega} \cos \phi \operatorname{sen}\left(\frac{\omega T}{2}\right) \cos \left(\theta+\frac{\omega T}{2}\right) \\
p_{y}+\frac{2 v}{\omega} \cos \phi \operatorname{sen}\left(\frac{\omega T}{2}\right) \operatorname{sen}\left(\theta+\frac{\omega T}{2}\right) \\
p_{z}+v \operatorname{sen} \phi \\
v \\
\theta+\omega T \\
\phi \\
\omega
\end{array}\right]_{k}
$$

O vetor de ruídos $\mathbf{w}_{k}$ mantém-se o mesmo para o caso bidimensional, porém a matriz $G_{k}$ tem a sua estrutura modificada para se adequar às dimensões do modelo:

$$
G_{k}=\left[\begin{array}{ccccccc}
0 & 0 & 0 & T^{2} & 0 & 0 & 0 \\
0 & 0 & 0 & 0 & \frac{T^{2}}{2} & 0 & T^{2}
\end{array}\right]^{\top} .
$$

\subsection{Modelo GC com Entrada Externa}

O modelo GC com entrada externa (GCEE) é o modelo proposto em (Kang et al., 2017). Assim como no modelo GCVP, apresentado na Seção 3.2, o modelo GCEE também lida com a velocidade, tomando como base seu módulo $v$ e seu ângulo de rumo $\theta$. No entanto, a velocidade angular $\omega$ não aparece mais como um estado de $\mathbf{x}_{k}$ e, sim, como um parâmetro de controle, assim como a aceleração $a$ do objeto.

Uma possível interpretação física desse modelo seria um cenário em que o usuário controla remotamente um objeto manobrante e este mesmo usuário tem a possibilidade de ajustar os parâmetros de aceleração e velocidade angular através desse controle.

Novamente, com base em (1), a equação do espaço de estados para o modelo dinâmico em questão pode ser reescrita da seguinte maneira:

$$
\mathbf{x}_{k}=F_{k-1} \mathbf{x}_{k-1}+B_{k-1} \mathbf{u}_{k-1}+G_{k-1} \mathbf{w}_{k-1},
$$

em que $B_{k}$ é a matriz de controle e

$$
\begin{aligned}
& \mathbf{x}_{k}=\left[\begin{array}{llll}
p_{x} & p_{y} & v & \theta
\end{array}\right]_{k}^{\top}, \\
& \mathbf{u}_{k}=[a \omega]_{k}^{\top} \text {, } \\
& \mathbf{w}_{k}=\left[\begin{array}{ll}
w_{v} & w_{\theta}
\end{array}\right]_{k}^{\top} .
\end{aligned}
$$

A matriz dinâmica $F_{k}$, por conta dos estados de $\mathbf{x}_{k}$, possui uma estrutura mais simplificada em relação à função apresentada em (13):

$$
F_{k}=\left[\begin{array}{cccc}
1 & 0 & T \operatorname{sen} \theta & 0 \\
0 & 1 & T \cos \theta & 0 \\
0 & 0 & 1 & 0 \\
0 & 0 & 0 & 1
\end{array}\right]_{k}
$$

Note que a matriz $F_{k}$ possui características não lineares, já que depende do estado $\theta$ através de funções trigonométricas. A matriz $G_{k}$, pelo fato do ruído afetar somente o módulo da velocidade $v$ e o ângulo de rumo $\theta$, fica estruturada da seguinte maneira:

$$
G_{k}=\left[\begin{array}{llll}
0 & 0 & 1 & 0 \\
0 & 0 & 0 & 1
\end{array}\right]^{\top}
$$

Por fim, a matriz de controle $B_{k}$, relativa ao vetor de controle $\mathbf{u}_{k}$, é dada por:

$$
B_{k}=\left[\begin{array}{llll}
0 & 0 & T & 0 \\
0 & 0 & 0 & T
\end{array}\right]^{\top} .
$$

Para a adaptação desse modelo para a sua versão tridimensional, alterações semelhantes às realizadas para o modelo GCVP são aplicadas. Dessa forma, serão adicionados ao vetor $\mathbf{x}_{k}$ dois novos estados: o ângulo de elevação em relação ao eixo $z, \phi$, e a coordenada da posição do objeto no eixo $z, p_{z}$. O vetor de estados fica, portanto, da seguinte maneira:

$$
\mathbf{x}_{k}=\left[\begin{array}{llllll}
p_{x} & p_{y} & p_{z} & v & \theta & \phi
\end{array}\right]_{k}^{\top}
$$

A matriz $F_{k}$ é obtida de forma similar à sua versão bidimensional e é apresentada em (26): 


$$
F_{k}=\left[\begin{array}{cccccc}
1 & 0 & 0 & T \cos \theta \cos \phi & 0 & 0 \\
0 & 1 & 0 & T \operatorname{sen} \theta \cos \phi & 0 & 0 \\
0 & 0 & 1 & T \operatorname{sen} \phi & 0 & 0 \\
0 & 0 & 0 & 1 & 0 & 0 \\
0 & 0 & 0 & 0 & 1 & 0 \\
0 & 0 & 0 & 0 & 0 & 1
\end{array}\right]_{k}
$$

Note que a matriz $F_{k}$ ainda possui características não lineares em relação a elementos que compõem o vetor de estados $\mathbf{x}_{k}$. A matriz de controle $B_{k}$ também sofre uma modificação, devido ao aumento do vetor de estados, e é dada por (27):

$$
B_{k}=\left[\begin{array}{llllll}
0 & 0 & 0 & T & 0 & 0 \\
0 & 0 & 0 & 0 & T & T
\end{array}\right]^{\top}
$$

Novamente, supõe-se que os ruídos do processo só afetam o módulo da velocidade $v$ e o ângulo de rumo $\theta$. Dessa forma, a matriz $G_{k}$ também sofre uma reestruturação, resultando em:

$$
G_{k}=\left[\begin{array}{llllll}
0 & 0 & 0 & 1 & 0 & 0 \\
0 & 0 & 0 & 0 & 1 & 0
\end{array}\right]^{\top}
$$

\section{FILTRO DE KALMAN ESTENDIDO}

Para verificar se os modelos apresentados nas Seções 3.13.3 são adequados para representar a dinâmica de um objeto manobrante, é necessário analisar a qualidade das estimativas de estado resultantes de um filtro estocástico, o qual utiliza tais modelos em suas equações de filtragem.

Observando tais modelos, é necessário um filtro estocástico que tenha em sua formulação o tratamento de modelos matemáticos não lineares. Dessa maneira, o filtro escolhido foi o filtro de Kalman estendido (FKE), que realiza linearizações, via série de Taylor, para aproximar as não linearidades em um dado ponto de operação.

Como todo filtro estocástico, o FKE possui equações de previsão, que determinam o valor do vetor de estados um passo à frente sem a informação de medida, e equações de atualização, que corrigem a previsão do passo anterior com a chegada de novas medidas.

As equações de previsão do FKE são as seguintes:

$$
\begin{aligned}
& \hat{\mathbf{x}}_{k \mid k-1}=\mathbf{f}\left(\hat{\mathbf{x}}_{k-1 \mid k-1}, \mathbf{u}_{k-1}\right), \\
& P_{k \mid k-1}=\mathcal{F}_{k-1} P_{k-1 \mid k-1} \mathcal{F}_{k-1}^{\top}+G_{k-1} Q_{k-1} G_{k-1}^{\boldsymbol{\top}},
\end{aligned}
$$

em que $\hat{\mathbf{x}}_{k \mid k-1}$ é o vetor de estados previsto, $\mathcal{F}_{k-1}$ é a matriz jacobiana relacionada a linearização da função $\mathbf{f}(\cdot)$ no ponto de operação $\hat{\mathbf{x}}_{k-1 \mid k-1}, Q_{k-1}$ é a matriz de covariância do ruído do processo e $P_{k \mid k-1}$ é a matriz de covariância do erro de previsão.

As equações de atualização são as seguintes:

$$
\begin{aligned}
\hat{\mathbf{x}}_{k \mid k} & =\hat{\mathbf{x}}_{k \mid k-1}+K_{k}\left(\mathbf{y}_{k}-\mathbf{g}\left(\hat{\mathbf{x}}_{k \mid k-1}\right)\right), \\
P_{k \mid k} & =P_{k \mid k-1}-K_{k} S_{k} K_{k}^{\top} \\
S_{k} & =\mathcal{G}_{k} P_{k \mid k-1} \mathcal{G}_{k}^{\top}+R_{k} \\
K_{k} & =P_{k \mid k-1} \mathcal{G}_{k}^{\top} S_{k}^{-1}
\end{aligned}
$$

em que $\hat{\mathbf{x}}_{k \mid k}$ é o vetor de estados atualizado, $P_{k \mid k}$ é a matriz de covariância do erro de estimativa, $S_{k}$ é a matriz de covariância da inovação, $K_{k}$ é o ganho de Kalman, $R_{k}$ é a matriz de covariância do ruído da medida e $\mathcal{G}_{k}$ é a matriz jacobiana relacionada a linearização da função $\mathbf{g}(\cdot)$ no ponto de operação $\hat{\mathbf{x}}_{k \mid k-1}$.

\section{SIMULAÇÕES E RESULTADOS}

Para realizar o comparativo de desempenho entre os modelos GCVP/GCEE reestruturados e o modelo GCVC, foram criadas duas trajetórias tridimensionais distintas, denominadas Cenário 1 e Cenário 2. Tais trajetórias foram criadas a partir do gerador de trajetórias proposto em (Martins et al., 2018) e são compostas por cinco trechos cada uma.

Para ambos os cenários, o FKE foi utilizado para obter as trajetórias estimadas. Os parâmetros relacionados às variâncias dos ruídos de processo, que compõem a matriz $Q_{k}$, são: $\sigma_{p Q}^{2}=1 \mathrm{~m}^{2}$ para cada posição, $\sigma_{v}^{2}=0,1(\mathrm{~m} / \mathrm{s})^{2}$ para velocidade, $\sigma_{\theta}^{2}=\sigma_{\phi}^{2}=0,0088 \mathrm{rad}^{2}$ para os ângulos de rumo e de elevação, respectivamente, e $\sigma_{\omega}^{2}=0,001(\mathrm{rad} / \mathrm{s})^{2}$ para a velocidade angular. Já os parâmetros relacionados às variâncias dos ruídos de medida, que compõem a matriz $R_{k}$, são: $\sigma_{p R}^{2}=4 \mathrm{~m}^{2}$ para cada posição.

Em seguida, além da obtenção do tempo de execução do código $t_{\text {exec }}$, foram calculados os EQMs para cada uma das coordenadas de posição: $\mathrm{EQM}_{x}, \mathrm{EQM}_{y}$ e EQM $\mathrm{EQ}_{z}$. O EQM para cada coordenada $\ell=\{x, y, z\}$ é calculado da seguinte forma:

$$
\operatorname{EQM}_{\ell}=\frac{1}{N} \sum_{k=1}^{N}\left(p_{\ell}(k)-\hat{p}_{\ell}(k)\right)^{2},
$$

em que $p_{\ell}(k)$ é a posição exata na direção da coordenada $\ell$ no instante de tempo $k$, fornecida pelo gerador de trajetórias, $\hat{p}_{\ell}(k)$ é a posição estimada na direção da coordenada $\ell$ no instante de tempo $k$, calculada pelo FKE, e $N$ é o número total de estimativas.

Com o intuito de facilitar a análise comparativa de resultados, criou-se um vetor EQM, cujos elementos são os EQMs de posição, ou seja:

$$
\mathrm{EQM}=\left[\begin{array}{lll}
\mathrm{EQM}_{x} & \mathrm{EQM}_{y} & \mathrm{EQM}_{z}
\end{array}\right]
$$

e, a partir de (36), calcula-se a sua norma vetorial $\|\mathrm{EQM}\|$.

\subsection{Cenário 1}

Os parâmetros de inicialização utilizados para criar a trajetória do Cenário 1 foram os seguintes: para posição inicial, $p_{x}^{0}=p_{y}^{0}=p_{z}^{0}=30 \mathrm{~m}$; para velocidade inicial, $v_{x}^{0}=$ $1 \mathrm{~m} / \mathrm{s}, v_{y}^{0}=0 \mathrm{~m} / \mathrm{s}$ e $v_{z}^{0}=1 \mathrm{~m} / \mathrm{s}$. As acelerações tangenciais $\left(a_{t}\right)$ e normais $\left(a_{n}\right)$ para cada trecho da trajetória são apresentadas na Tabela 1 e os valores de aceleração $a$ e velocidade angular $\omega$, relacionados ao vetor de controle do modelo GCEE, são apresentados na Tabela 2.

Tabela 1. Acelerações por Trecho - Cenário 1.

\begin{tabular}{|c|c|c|c|c|c|}
\hline \hline Trecho & 1 & 2 & 3 & 4 & 5 \\
\hline \hline$a_{t}\left(\mathrm{~m} / \mathrm{s}^{2}\right)$ & 2 & 1 & 4 & 6 & 4 \\
\hline$a_{n}\left(\mathrm{~m} / \mathrm{s}^{2}\right)$ & 0 & $-0,7$ & 1 & 0,7 & $-0,1$ \\
\hline \hline
\end{tabular}

Tabela 2. Parâmetros de Controle - Cenário 1.

\begin{tabular}{|c|c|c|c|c|c|}
\hline \hline Trecho & 1 & 2 & 3 & 4 & 5 \\
\hline$a\left(\mathrm{~m} / \mathrm{s}^{2}\right)$ & 2 & 1,221 & 4,123 & 6,041 & 4,001 \\
\hline$\omega(\mathrm{rad} / \mathrm{s})$ & 0 & $-0,031$ & 0,032 & 0,016 & $-0,002$ \\
\hline \hline
\end{tabular}


Na Figura 1, tem-se a trajetória do Cenário 1, sendo que a Figura 2 é a projeção desta mesma trajetória no plano $x y$, com o intuito de facilitar a visualização da trajetória estimada.

Em ambas as figuras, são apresentadas a trajetória exata (preto), a trajetória estimada pelo FKE (azul) com o modelo GCVP e as medidas ruidosas (pontos em vermelho), obtidas pelo gerador de trajetórias. Pelo fato das trajetórias estimadas com os outros modelos serem visualmente semelhantes à obtida com o modelo GCVP, optou-se por apresentar apenas as trajetórias para este modelo.

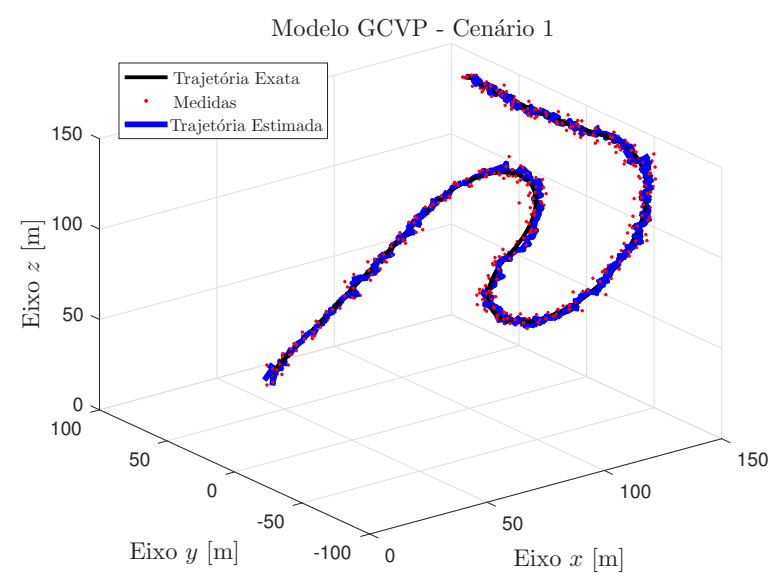

Figura 1. Cen. 1 - Visão 3D - Estimativas (GCVP).

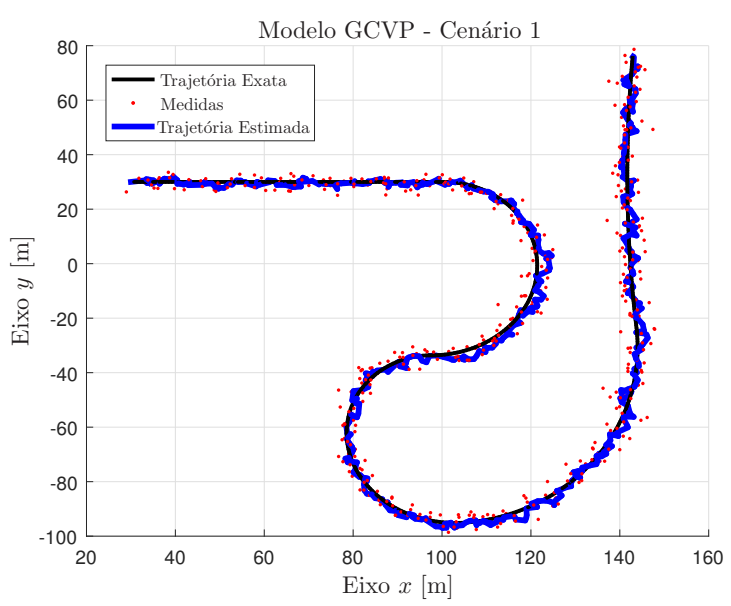

Figura 2. Cen. 1 - Visão 2D - Estimativas (GCVP).

De forma qualitativa, é possível observar que a trajetória estimada pelo FKE permanece sempre entre as medidas ruidosas, indicando que houve uma redução da influência dos ruídos dessas medidas na trajetória estimada.

Sob o ponto de vista quantitativo, foram calculadas as normas do vetor EQM e obtidos os tempos de execução de código $t_{\text {exec }}$ (em segundos) para cada um dos modelos tridimensionais apresentados neste trabalho. Tais valores encontram-se na Tabela 3.
Tabela 3. Cenário 1 - ||EQM $\|$ e $t_{\text {exec }}$.

\begin{tabular}{|c|c|c|c|c|}
\hline \hline Modelo & $\|\mathrm{EQM}\|$ & Redução EQM & $t_{\text {exec }}$ & Redução $t_{\text {exec }}$ \\
\hline \hline GCVC & 2,6932 & - & 0,1369 & - \\
\hline GCEE & 2,6420 & $1,9 \%$ & 0,0906 & $33,8 \%$ \\
\hline GCVP & 2,5371 & $5,7 \%$ & 0,1302 & $4,8 \%$ \\
\hline
\end{tabular}

Note que os valores de $\|\mathrm{EQM}\|$ dos modelos propostos atingem um valor menor do que para o modelo GCVC, comprovado pela porcentagem de redução do $\|\mathrm{EQM}\|$ do modelo GCVC. O tempo de execução para os modelos propostos também são inferiores ao do modelo GCVC, sendo a maior redução do $t_{\text {exec }}$ ocorrida no modelo GCEE.

\subsection{Cenário 2}

Os parâmetros utilizados para criar a trajetória do Cenário 2 foram os seguintes: para posição inicial $p_{x}^{0}=p_{y}^{0}=p_{z}^{0}=$ $10 \mathrm{~m}$; para velocidade inicial $v_{x}^{0}=0 \mathrm{~m} / \mathrm{s}, v_{y}^{0}=1 \mathrm{~m} / \mathrm{s}$ e $v_{z}^{0}=1 \mathrm{~m} / \mathrm{s}$. As acelerações tangenciais e normais para cada um dos trechos são apresentadas na Tabela 4 e os valores de aceleração e velocidade angular, definidos para o vetor de controle do modelo GCEE, são apresentados na Tabela 5.

Tabela 4. Acelerações por Trecho - Cenário 2.

\begin{tabular}{|c|c|c|c|c|c|}
\hline \hline Trecho & 1 & 2 & 3 & 4 & 5 \\
\hline \hline$a_{t}\left(\mathrm{~m} / \mathrm{s}^{2}\right)$ & 4 & 7 & 5 & 6 & 4 \\
\hline$a_{n}\left(\mathrm{~m} / \mathrm{s}^{2}\right)$ & 0,1 & $-0,7$ & 1 & $-1,4$ & $-1,4$ \\
\hline \hline
\end{tabular}

Tabela 5. Parâmetros de Controle - Cenário 2.

\begin{tabular}{|c|c|c|c|c|c|}
\hline \hline Trecho & 1 & 2 & 3 & 4 & 5 \\
\hline \hline$a\left(\mathrm{~m} / \mathrm{s}^{2}\right)$ & 4,001 & 7,034 & 5,099 & 6,161 & 4,238 \\
\hline$\omega(\mathrm{rad} / \mathrm{s})$ & 0,007 & $-0,018$ & 0,019 & $-0,023$ & $-0,020$ \\
\hline \hline
\end{tabular}

Na Figura 3, o Cenário 2 é ilustrado, sendo a Figura 4 a projeção da respectiva trajetória tridimensional no plano $x y$, para facilitar a visualização da trajetória estimada em relação à trajetória exata e às medidas.

Novamente, a trajetória exata aparece em preto, a trajetória estimada pelo FKE com o modelo GCVP, está em azul e as medidas ruidosas são representadas por pontos avermelhados. Pelo fato das outras trajetórias estimadas serem visualmente semelhantes à obtida com o modelo GCVP, optou-se por apresentar apenas as trajetórias para este modelo.

Qualitativamente, é possível notar que a trajetória estimada permanece próxima da trajetória exata, sempre dentro da região de espalhamento das medidas, mostrando uma diminuição da influência do ruído nas estimativas.

De maneira semelhante ao feito para o Cenário 1 , foram calculadas as normas do vetor EQM e obtido o tempo de execução, em segundos, relacionado a cada um dos modelos dinâmicos apresentados aqui, de forma a realizar uma análise quantitativa de desempenho. As normas de EQM e o tempo de execução para o Cenário 2 encontramse na Tabela 6 . 
Modelo GCVP - Cenário 2

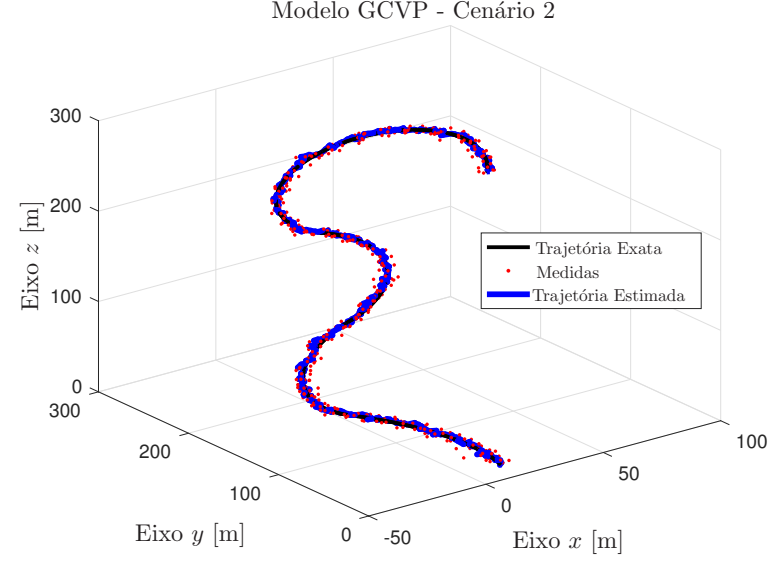

Figura 3. Cen. 2 - Visão 3D - Estimativas (GCVP).

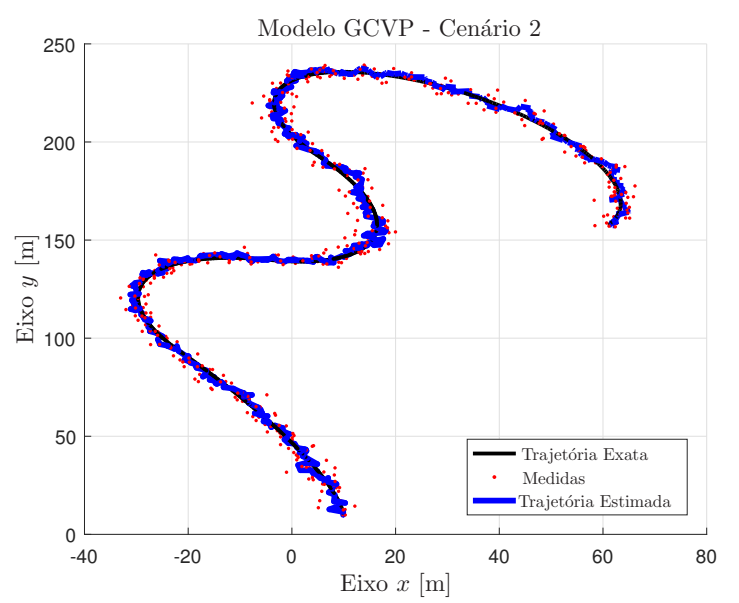

Figura 4. Cen. 2 - Visão 2D - Estimativas (GCVP).

Tabela 6. Cenário $2-\|\mathrm{EQM}\|$ e $t_{\text {exec }}$.

\begin{tabular}{|c|c|c|c|c|}
\hline \hline Modelo & $\|\mathrm{EQM}\|$ & Redução EQM & $t_{\text {exec }}$ & Redução $t_{\text {exec }}$ \\
\hline \hline GCVC & 2,7368 & - & 0,1377 & - \\
\hline GCEE & 2,5761 & $5,8 \%$ & 0,0932 & $32,3 \%$ \\
\hline GCVP & 2,2931 & $16,2 \%$ & 0,1212 & $11,9 \%$ \\
\hline \hline
\end{tabular}

Novamente, os valores de $\|\mathrm{EQM}\|$, relacionados aos modelos propostos, atingem um valor menor do que o obtido para o modelo GCVC, comprovado mais uma vez pela redução do erro. Desta vez, a diminuição do erro é mais perceptível, chegando a uma redução de 16,2\%. A redução do $t_{\text {exec }}$ também pode ser considerada significativa, com o modelo GCEE novamente obtendo a maior redução de valor.

\section{CONCLUSÃO}

Este trabalho teve como objetivo reestruturar modelos dinâmicos bidimensionais encontrados na literatura, de tal maneira a fazer com que estes modelos passassem a representar trajetórias tridimensionais de objetos manobrantes. Estes modelos dinâmicos, que se baseiam em coordenadas polares para definir a velocidade do objeto, foram comparados com outro modelo tridimensional, porém com a velocidade definida em coordenadas cartesianas. Para verificar se os modelos dinâmicos propostos são adequados, tais modelos foram implementados computacionalmente em um FKE.

A partir das trajetórias estimadas pelo FKE, foram feitas análises qualitativas e quantitativas dos resultados obtidos. Pôde-se verificar que os modelos propostos são adequados para descrever a dinâmica de um objeto manobrante no espaço, apresentando bons índices de desempenho, com EQM e $t_{\text {exec }}$ menores do que o modelo escolhido como referencial de comparação, o modelo GCVC.

Os próximos passos estão concentrados em aplicar os modelos propostos em um filtro IMM (Rong Li and Jilkov, 2005), que é composto por um banco de filtros estocásticos que chaveiam probabilisticamente entre si, priorizando o modelo dinâmico mais adequado para o instante de tempo atual.

\section{AGRADECIMENTOS}

Os autores agradecem o apoio do Departamento Acadêmico de Eletrotécnica - DAELT, da Universidade Tecnológica Federal do Paraná - UTFPR e o Projeto Universal - CNPq Processo: 421486/2016-3 pelo suporte financeiro.

\section{REFERÊNCIAS}

Bar-Shalom, Y., Rong Li, X., and Kirubarajan, T. (2001). Estimation with applications to tracking and navigation: theory algorithms and software. John Wiley \& Sons.

Frencl, V.B., do Val, J.B.R., and Mendes, R.S. (2008). Filtragem Utilizando IMM-EKF-BLUE em Modelos de Giro Constante com Taxa de Giro Desconhecida. In XVII Congresso Brasileiro de Automática (CBA 2008). Juiz de Fora, MG.

Gustafsson, F. and Isaksson, A.J. (1996). Best choice of coordinate system for tracking coordinated turns. In Proceedings of 35th IEEE Conference on Decision and Control, volume 3, 3145-3150.

Jazwinski, A.H. (1970). Stochastic processes and filtering theory. Academic Press.

Kang, M., Liu, Y., and Zhao, Y. (2017). A threat modeling method based on kalman filter for uav path planning. In 2017 29th Chinese Control And Decision Conference $(C C D C), 3823-3828$.

Martins, G.D.M., Naruto, I.L., Danner, P., and Frencl, V.B. (2018). A trajectory simulator using frenet-serret formulas applied to punctual objects. In 2018 13th IEEE International Conference on Industry Applications (INDUSCON), 750-755.

Ogata, K. (2015). Engenharia de Controle Moderno. Pearson, 5ed.

Rong Li, X. and Jilkov, V.P. (2003). Survey of maneuvering target tracking. part i. dynamic models. IEEE Transactions on Aerospace and Electronic Systems, 39(4), 1333-1364.

Rong Li, X. and Jilkov, V.P. (2005). Survey of maneuvering target tracking. part v. multiple-model methods. IEEE Transactions on Aerospace and Electronic Systems, 41(4), 1255-1321. 